\title{
Threat learning impairs subsequent memory recombination with past episodes
}

\author{
Olivier T. de Vries ${ }^{1}$, Raoul P.P.P. Grasman ${ }^{2}$, Merel Kindt ${ }^{1,3}$, Vanessa A. van Ast ${ }^{1,3}$ \\ ${ }^{1}$ Department of Clinical Psychology, University of Amsterdam, Amsterdam, the Netherlands \\ ${ }^{2}$ Department of Psychological Methods, University of Amsterdam, Amsterdam, the Netherlands \\ ${ }^{3}$ Amsterdam Brain and Cognition, University of Amsterdam, Amsterdam, the Netherlands
}

Please address correspondence to:

Olivier T. de Vries (o.t.devries@uva.nl) or Vanessa A. van Ast (V.A.vanAst@uva.nl)

Faculty of Social and Behavioral Sciences, Department of Clinical Psychology

Nieuwe Achtergracht 129

1018 WS Amsterdam 


\begin{abstract}
To successfully predict important events, the representations in memory on which we rely need to be constantly updated and transformed to best reflect a complex and dynamic world. Here we employed a novel paradigm to investigate how memories of threat learning affect the flexible recombination across distinct but overlapping experiences, an ability referred to as relational memory. Participants $(n=35)$ visited the lab to first encode neutral associations $(A \rightarrow$ B), which were reactivated and predictively associated with a new aversive or neutral element ( $B \rightarrow C_{\text {ThREAT/NeUtRAL }}$ ) on the following day, whilst pupil dilation was measured as an index of arousal. Then, again one day later, the accuracy of relational memory judgements $(A \rightarrow C$ ?) was tested. Novel association to threat was found to impair relational memory. Unexpectedly, this effect was not moderated by arousal. We propose that compartmentalization of threat learning events could be a function of a healthy memory, preventing maladaptive 'episodic overgeneralization' of threat to previously encoded episodes.
\end{abstract}

Keywords: Relational memory, integration, threat learning, arousal 


\section{Introduction}

The last two decades have seen a dramatic shift in how episodic memories are understood, from rigid, passive records of the past to flexible, actively constructed representations in service of the future (Klein et al., 2002; Schacter et al., 2007). This conceptual shift is underlined by the recent surge in studies investigating relational memory and the neurocognitive mechanisms that afford it (Zeithamova et al., 2012). Relational memory refers to our ability to recombine information across distinct episodes (Eichenbaum, 1999), and functions to inform us in novel situations when habits and memories of single experiences are insufficient to generate the predictions that are crucial to decision making (Biderman et al., 2020). From an evolutionary point of view, our most valuable memories are those that help us predict aversive experiences that we would wish to avoid. However, despite the central role that negatively arousing memories are thought to play in guiding adaptive future behavior (LaBar \& Cabeza, 2006), studies investigating relational memory have neither explored emotional memory, nor accounted for its fundamentally prospective purpose. It is thus presently unknown how relational memory is affected when a premise memory constitutes a predictive threat learning experience, and whether such an effect is driven by arousal.

Tolman (1948) was the first to suggest that overlapping memory traces can be stored as interconnected relational structures which he dubbed 'cognitive maps'. Contemporary neuroscientific studies demonstrate that memories from distinct experiences that share a common element are indeed integrated at the representational level (Schlichting et al., 2015; Shohamy \& Wagner, 2008). Importantly, these findings imply that under certain conditions the representations that facilitate subsequent relational memory are established before they are 
actually required (Shohamy \& Daw, 2015). Under the hypothesis that the construction of those representations which are likely to guide future decisions that are of biological importance are prioritized over those that are not, relational memory for threatening events may be enhanced. Emotional enhancements have previously been shown for neutral memories that proved relevant only after initial encoding. For example, recognition memory has been shown to be enhanced for items that, through new emotional learning, have acquired some predictive value as a semantic category (Dunsmoor et al., 2015). As for associative memories, Zhu et al. (2020) recently revealed that when one element of an existing memory is paired to an emotional stimulus, associations between elements of that original memory are strengthened. Similar evidence comes from a study using monetary reward as a reinforcing stimulus, showing that a hippocampus-dependent mechanism allows positive value to spread to older, indirectly related memories that are reactivated during reinforcement learning, biasing subsequent decision making (Wimmer \& Shohamy, 2012). However, note that in these studies it was not possible to explicitly test the associations across memories between reactivated items and novel emotional stimuli (i.e., relational memory), since the latter (shock or monetary reward) were not episodic in nature, nor unique to each episode.

In sharp contrast with this hypothesis that relational memory is enhanced when one premise memory involves a threat learning experience, research to the effects of negative emotion on simple associative memory paints a different picture. Memory for associations between items is typically impaired when a negative stimulus is involved (Chiu et al., 2013). This effect is thought to be driven primarily by noradrenergic arousal (Guez et al., 2015). As a mechanistic explanation dubbed the disruption hypothesis, Bisby, Horner, \& Bush (2018) have 
proposed that an emotionally negative element as part of an episodic association decreases the likelihood of successful pattern completion, an effect presumably resulting from arousalinduced reductions in hippocampal activity (Bisby et al., 2016). Given that the representations required for relational memory judgements are particularly dependent on hippocampal processing, even more so than within-memory associations (Zeithamova et al., 2012), they may be especially sensitive to disruptions due to amygdala-induced noradrenergic arousal (McGaugh, 2004). An alternative explanation, the bypassing hypothesis, posits that emotional associations are impaired because these cannot benefit, like neutral associations do, from extrahippocampal areas involved in unitizing memory elements, but are dependent only on hippocampal processing (Madan et al., 2017). Though neither of these hypotheses make explicit predictions regarding relational memory, both of the impairing mechanisms proposed could extend to the formation of associations between emotional experiences and reactivated memories.

Here we reasoned that predictive threat learning can, once consolidated, affect relational memory. However, since opposing hypotheses regarding the direction of this effect can be derived from the literature, this was left open. We further hypothesized that, regardless of the direction, the magnitude of the effect is moderated by noradrenergic arousal responses during threat learning. To test whether and how biologically relevant threat learning impacts flexible recombination across memories, we developed the 'Predictive Relational Emotional Memory (PRE-Memory) paradigm', for which participants visited the lab on three consecutive days. The decision to spread out learning and testing over several days was motivated by reasons pertaining to both synaptic and systems consolidation: First, the effects of emotion on episodic 
memory typically require time to emerge (McGaugh, 2018), as alteration via synaptic consolidation is a protein-synthesis depended process (Hernandez \& Abel, 2009). Second, the formation of cognitive-map like overlapping representations and extraction of regularities from them has always been thought of as time-dependent (Ellenbogen et al., 2007; McClelland et al., 1995). Unlike simultaneous presentation of neutral and emotional pictures, the here implemented episodic threat learning procedure is likely to elicit defensive preparations to actively predict and cope with an impending emotional state, triggering motivational systems for future-oriented action (Krypotos et al., 2014). On the first day participants encoded neutral premise memories $(A \rightarrow B)$, which were predictively linked to multimodal, negative arousing or neutral control stimulus ( $\mathrm{B} \rightarrow \mathrm{C}_{\text {THREAT/NEUTRAL }}$ ) on the following day. Noradrenergic arousal responses to $B$ and $C$ items during episodic threat learning were indexed by means of pupillometry. On the third and final day participants made all possible relational memory judgements $\left(A \rightarrow C_{\text {ThREAT/NeUtRAL }}\right.$ ). Employing a novel paradigm, the present study sheds light on the functioning of a complex feature of episodic memory in those situations when it may matter the most. 


\section{Methods}

\subsection{Participants}

Forty-seven healthy individuals were recruited via the university's online system and gave written consent to participate in this study. Exclusion criteria as assessed via a screening based on self-report were recreational drug use at a frequency above once a month, average consumption of 21 or more units of alcohol per week, having experienced trauma, and having received treatment for a mental disorder listed in the DSM-5 by a psychologist or psychiatrist in the past year. Participants were rewarded either with course credits or 45 euros for completing the experiment, or according to the total amount of time spent in the lab in case of dropping out. Due to the high aversiveness of the picture-sound combinations on day two, it was emphasized that they were free to quit the experiment at any time without having to give a reason. Ten participants made use of this option on day two. Additionally, one participant aborted the computer task on day two by accidentally pressing the escape button, and another missed the appointment for the third day. The final sample thus includes data of 35 participants (mean age $=21.4, \mathrm{SD}=2.6 ; 24$ women). This sample size is more than sufficient to give reliable parameter estimates in a two-level multilevel model (Maas \& Hox, 2015). For analyses using pupil data, an additional three were excluded for not consistently reaching the data quality criteria. This study was approved by the local ethics committee of the University of Amsterdam. 


\subsection{Materials}

\subsubsection{Stimuli}

We selected 80 neutral pictures of objects from the Bank of Standardized Stimuli (BOSS;

(Brodeur et al., 2010) to function as A and B stimuli. Forty C stimuli, 20 emotionally negative and 20 neutral, were partly chosen from the Nencki Affective Picture System (NAPS)

(Marchewka et al., 2014), and supplemented with copyright-free photos from the internet. Each $\mathrm{C}$ stimulus in the threat condition was matched to one in the neutral condition in terms of its content, to control for memory effects of complexity and semantics. For example, an image of a fatal car crash in the threat condition was matched with an image of a car safely driving on the freeway in the neutral condition. For each $\mathrm{C}$ stimulus a corresponding sound was found from either the International Affective Digitized Sounds (IADS) database (Stevenson, Ryan A ; James, 2008), or The Freesound Project; a collaborative, open-source repository of audio content under a creative commons license (https://freesound.org/). These were selected to meaningfully match the image (e.g., image of badly broken leg - sound of snapping celery) to better emulate a real-life, multi-modal experience, thus enhancing the potential of aversive stimuli to evoke an ecologically valid physiological defensive response and corresponding associative memory.

Two important adjustments were made to the stimuli. First, the mean luminance of all images was set equal using the color adaptation of the SHINE toolbox for MATLAB. This diminishes the potential influence of low-level visual features of the stimuli that may confound pupil data (Willenbockel et al., 2010). Second, the maximum amplitude of each sound file was set such that it never exceeded 72 decibels in our lab setting, so none of the pupil effects across conditions could be explained as the result of loud noises (Liao et al., 2016). 


\subsubsection{Experimental Tasks}

The different phases of the PRE-Memory paradigm mirror the three distinct phases of a sensory preconditioning experiment (Brogden, 1947), and took place on three consecutive days. First, neutral predictive associations are learned between pairs of stimuli $(A \rightarrow B)$. Then, of each pair, one (B) is used as a conditioned stimulus (CS) for associative learning, with each either neutral or aversive $C$ item functioning as a unique, episodic, unconditioned stimulus (US; $B \rightarrow C$ ). Finally, we measure participants ability to recombine information from both learning days by presenting each $A$ item from day 1 , which has gained indirect predictive value for an either neutral or aversive $C$ item on day $2(A \rightarrow C$ ?). The experiment was programmed such that each participant was shown uniquely randomized combinations of $A \rightarrow B \rightarrow C$ stimuli.

\subsubsection{Learning (day 1)}

On the first day participants were shown 40 pairs $(A \rightarrow B)$ of sequentially presented pictures. Each trial started with a fixation cross presented for $500 \mathrm{~ms}$, after which stimulus A was shown for $4 \mathrm{~s}$, immediately followed by stimulus B for another $4 \mathrm{~s}$. Intertrial intervals (ITI) randomly varied between $8,9,10,11$, or $12 \mathrm{~s}$, with an average of $10 \mathrm{~s}$. Following presentation of all 40 unique pairs, they were all presented again in randomized order during a second and third learning block. Participants were given one-minute breaks between learning blocks. In the third block they were asked to indicate the vividness of each of their imagined stories on a visual analogue scale (VAS). 


\subsubsection{Learning (day 2)}

The associative learning task of day two was almost identical in structure and instructions to that of the previous day, but very different in terms of the presented stimuli. First, the stimuli presented were now $B \rightarrow C$ pairs, meaning that each first image presented $(B)$ was the second of a pair seen the day before. Moreover, the second item of each pair (C) could either be neutral or highly aversive (threat), and was accompanied by a corresponding sound played through headphones. This way, half of the $40 \mathrm{~A} \rightarrow \mathrm{B}$ pairs from the first day were 'extended' with a highly aversive $\mathrm{C}$ item $\left(\mathrm{A} \rightarrow \mathrm{B} \rightarrow \mathrm{C}_{\text {THREAT }}\right.$ ), whereas the other half were newly associated with a neutral control $\mathrm{C}$ item $\left(\mathrm{A} \rightarrow \mathrm{B} \rightarrow \mathrm{C}_{\text {NEUTRAL }}\right.$ ). As on the first day, after presentation of all $40 \mathrm{~B} \rightarrow \mathrm{C}$ pairs, these were repeated in a second and third learning block. Additionally, in the first learning block participants were asked to indicate the valence and arousal induced by the stories they imagined with each pair of items on two separate VASs. ITI duration and randomization were the same as the previous day, and participants were again given 1-minute breaks between learning blocks. A maximum of three $B \rightarrow C$ pairs from the same condition were presented consecutively.

\subsubsection{Relational memory test (day 3)}

To probe predictive reactivation during test, each A item was presented for four $\mathrm{s}$ followed by a self-paced, six-alternative forced-choice (6-AFC) test in which participants had to select the $C$ item it is indirectly associated with through a shared B item. Participants would use the numbers one to six on the numpad to indicate their answer for each trial. All of the lures presented during each test trial were other $\mathrm{C}$ stimuli that have been presented during the 
experiment and selected such that three were from the neutral condition and three from the threat condition. All C pictures were used equally often as lures.

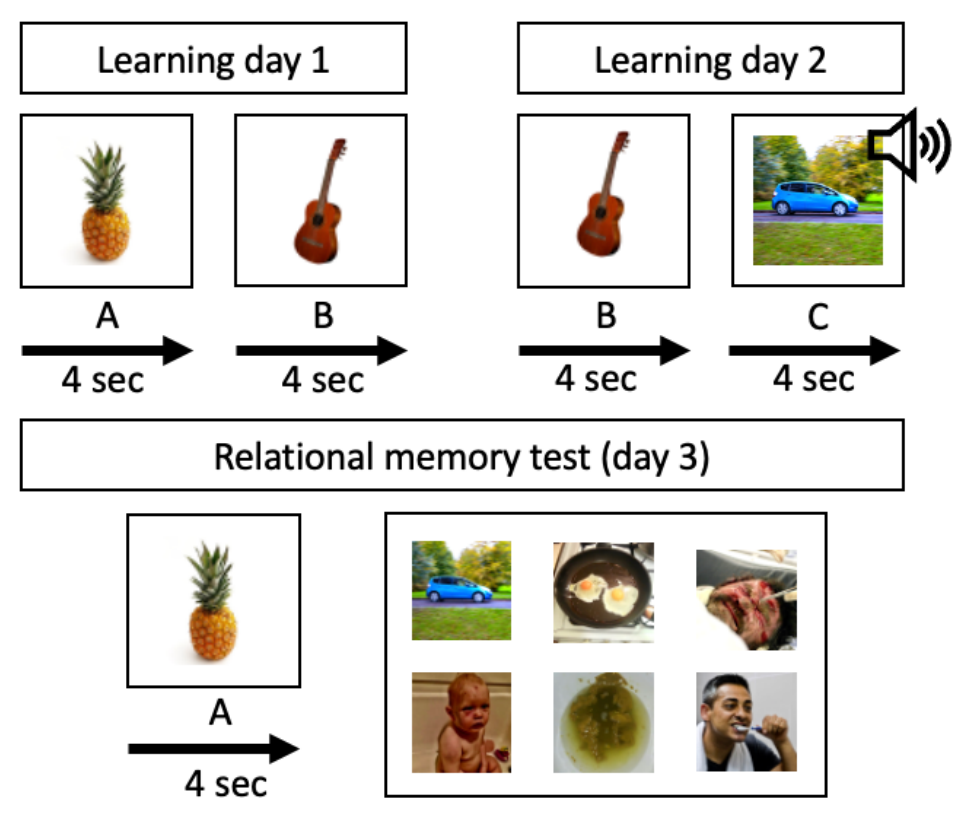

Fig 1. Overview of task structure on both learning days and the relational memory test phase on day 3. On the first day participants imagined stories in which they themselves play a central role on the base of pairs of sequentially presented neutral images. In this example the pineapple (A) is presented first, followed by the guitar (B). Then on day 2, each B item is presented and followed by a picture/sound combination (C) that can either be neutral or aversive. Participants were again instructed to vividly imagine stories. All pairs on both day 1 and 2 are presented a total of 3 times. During the last block of pair presentations on each day participants are asked to indicate the vividness of their imagined story for each pair. Finally, during the relational memory test, $\mathrm{A}$ items are presented followed by a six-alternative forced-choice test in which the correct $\mathrm{C}$ item must be selected using the numpad on the keyboard.

\subsubsection{Associative recognition tests (day 1, 2, \& 3)}

Over the course of the study, a total of four associative recognition tests were performed by each participant. First after learning on day 1 and after learning on day 2, and again following 
the relational memory test to measure which of the direct $A \rightarrow B$ and $B \rightarrow C$ associations were still accurately retained on day three. Similar to the relational memory test, a cue would be presented for four seconds after which participants had to select its associated stimulus out of six options from the same stimulus category ( $\mathrm{B}^{\prime} \mathrm{s}$ or $\mathrm{C}^{\prime} \mathrm{s}$ ), again balanced across conditions. All trials were self-paced.

\subsection{Procedure}

Each session took place in the same lab room where the lighting was kept at the maximum level so baseline pupil diameters would be low, leaving room for dilation in response to aversive stimuli.

\subsubsection{Day 1}

On the first day participants were informed that the goal of the experiment is to study their ability to vividly imagine stories involving different picture combinations. This was to obscure the fact that the primary interest was memory, and so prevent the use of deliberate and variable learning strategies. After reading the information brochure and signing an informed consent form, participants took place in front of the computer screen. The experimenter read out the instructions for both the first associative learning task under the guise of an 'imagination task', and the associative recognition test that would follow. Specifically, participants were instructed to use each pair of presented stimuli to vividly imagine stories in which they themselves play a central role. Instructions for both tasks were repeated on the 
computer screen, following tasks took place. At the end of the session a brief exit questionnaire was conducted inquiring after the participants motivation to comply with instructions.

\subsubsection{Day 2}

On the second day of the experiment participants again received verbal instructions for the associative learning task and associative recognition test, which were repeated on the screen. They were not explicitly told that each first picture of a pair (B) would be the second picture of a pair they were presented yesterday, nor to actively reactivate its pre-existing associate (A). Participants were further asked to rest their head in a chinrest, and to minimize head movements during the experimental tasks so as to not interfere with pupil measurements. The session ended with an exit questionnaire, which this time also included questions inquiring after the participants' subjective emotional experience of the task.

\subsubsection{Day 3}

On the final day participants first performed the relational memory test, followed by two associative recognition tests for the associations that had been learned on day one $(A \rightarrow B)$ and two $(B \rightarrow C)$ of the experiment. They received verbal and written instructions for each test separately. Finally, after the last exit questionnaire, again inquiring after their motivation to comply with the instructions of the tasks, participants were debriefed on the true objectives of the experiment. 


\subsection{Data analysis}

\subsubsection{Acquisition and pre-processing}

Behavioral data acquisition was performed using Presentation software (Neurobehavioral Systems Inc., Berkeley CA). Pupil data during day 2 was collected using a Tobii Pro Nano eye tracker set at a sampling rate of $60 \mathrm{~Hz}$. The resulting time series were preprocessed using the Python programming language by 1) locating all samples registered by the eyetracker as missing values ( $\mathrm{NaN}$ ) as a result of participants blinking, looking away, or technical errors, and setting the samples 100 ms before and after to also be $\mathrm{NaN}, 2$ ) linearly interpolating around these $\mathrm{NaN}$ values, and 3) applying a band-pass filter (0.01-6 Hz, third-order Butterworth).

Following these first steps, pupil responses were quantified for B and C stimuli by computing the mean value in the frame of interest: In case of the B stimuli, which here function as conditioned stimuli, this was the final second of presentation, as anticipatory fear-responses are most likely to be picked up just before the fear-invoking stimulus (Koenig et al., 2017). For C stimuli, we looked at the final $2 \mathrm{~s}$ of stimulus presentation, where the emotional response is at its peak (Bradley et al., 2008). For a trial value to be deemed of sufficient quality to be included in the analyses, both the mean value for a frame of interest and its corresponding baseline had to be computed on the basis of at least $50 \%$ non-NaN values, or otherwise it was set to missing. The mean values for each frame of interested were subtracted from the mean pupil width during a baseline of 500 ms before B onset. Participants for whom over $50 \%$ of trials in either condition were excluded based on this criterion were excluded from the analyses altogether. 


\subsubsection{Manipulation and premise checks}

\subsubsection{Premise associations}

Following the relational memory test $(A \rightarrow C)$ on day 3 of the experiment, participants completed associative recognition tests for both premise memories $A \rightarrow B$ and $B \rightarrow C$, allowing for the specific selection of those $A \rightarrow C$ test trials for which the memories on which they are based have been retained (Zeithamova et al., 2013). Whether this resulted in an equal distribution of trials across conditions was assessed by means of an independent t-test. Furthermore, we assessed differences in associative memory between conditions immediately following learning on day one and two, also by means of independent t-tests.

\subsubsection{Arousal responses to aversive stimuli and transfer to predictors}

Two Condition (threat, neutral) $\times$ Block (one, two, three) repeated measures ANOVAs were carried out to test whether the aversive $\mathrm{C}$ stimuli were successful in evoking an arousal response, and whether these carried over to the $B$ stimuli in the second and third block following learning. For the former, average responses to the $\mathrm{C}$ stimuli were inserted as dependent variable, while for the latter, average pupil responses to B stimuli were used.

\subsubsection{Primary analyses}

We employed multilevel regression analyses, a modelling strategy that allows for hypothesis testing at the level of individual memories whilst taking into account the nested structure of memory trials within participants, for each of the main research questions posed here. As the dependent variable of each analysis is the binary outcome of the relational memory test trials 
which can either be correct or incorrect, we ran logistic multilevel regression models to test each hypothesis. All continuous predictor variables were group-mean centered, meaning that results can be interpreted as effects of within-subject predictor variance.

Importantly, all the trials analyzed here were only those for which both premise associations $(A \rightarrow B$ and $B \rightarrow C$ ) were retained on the day of relational memory testing. This means that the results are to be interpreted as the effects of threat learning, arousal, and encoding vividness in those instances that the memories required to make an $A \rightarrow C$ judgement are readily available.

\subsubsection{Effect of threat learning on relational memory}

To test the hypothesis that threat learning affects future relational memory, we ran a simple multilevel logistic regression model with condition as the only predictor variable, where the predicted mean response accuracy (odds_hit) for trial $j$, nested in participant $i$, is:

$$
\text { Odds_hit } t_{i j}=\mu+\alpha_{i}+\beta_{1}\left(\text { Threat }_{\mathrm{ij}}\right.
$$

The neutral condition was set as the reference category, meaning that the intercept $\mu$, or grand mean, can be interpreted as the predicted average odds for making a correct relational memory judgement for this condition. Individual variance in baseline performance, or likelihood of accurate relational memory in the neutral condition, is captured by the random intercepts, $\alpha_{i}$.

The coefficient $\beta_{1}$, then represents the difference in predicted average odds for threat trials relative to neutral trials. If the $\beta_{1}$ parameter is significant, this indicates an effect of threat 
learning. A positive value for $\beta_{1}$ would be evidence for an enhancing effect of threat, whereas a negative value would suggest an impairment.

\subsubsection{Noradrenergic moderation of threat learning's effect on relational memory}

Then, to assess whether arousal evoked by the aversive $C$ stimuli (i.e., the US stimuli) was driving the possible effect of threat memories on relational memory (be it enhancement or impairment), pupil responses upon first encounter of $C$ in the first block were added as moderators of the effect of condition:

$$
\text { Odds_hit } t_{i j}=\mu+\alpha_{i}+\beta_{1}(\text { Threat })_{i j}+\beta_{2}(\text { Arousal })_{i j}+\beta_{3}(\text { Threat } \times \text { Arousal })_{i j}
$$

Since arousal, operationalized as pupil dilation, is a continuous variable, $\beta_{2}$ represents the corresponding slope for predicting the odds ratio. The neutral condition is again reference, meaning that $\beta_{3}$, the slope of the arousal $\mathrm{x}$ condition interaction, represents the difference in slopes for the effect of arousal in threat trials, relative to neutral trials.

\subsubsection{Secondary analyses}

\subsubsection{Controlling for vividness of premise memories}

The extent by which threat learning will affect relational memory may depend on the subjective vividness of the original episodes that form the basis for a subsequent judgement. Emotionally arousing events are well-known to produce vivid memories (Todd et al., 2012), which in turn are linked to enhanced associations (Murray \& Kensinger, 2012, 2013). Thus, to control for effects of 
premise memory vividness, which may differ across conditions, participants gave a subjective vividness rating for premise memories on both learning days.

We tested whether vividness moderates the effect of threat learning on subsequent relational memory by adding the vividness score for day 1 and day 2 to the model, specified as a threeway interaction with condition:

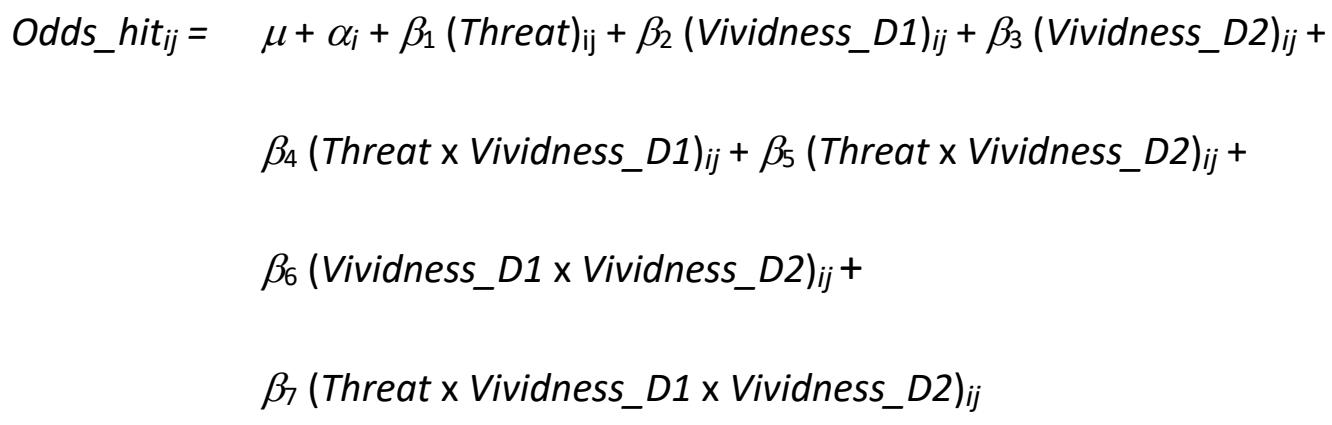

The vividness variables for both days, Vividness_D1 and Vividness_D2, are continuous variables, their respective main effects indicated by slopes $\beta_{2}$ and $\beta_{3}$. As is the case in the previous analyses, the interaction effects $\beta_{4}$ and $\beta_{5}$ indicated the differences in the effect of premise memory vividness for threat trials relative to neutral trials for both encoding days. Finally, parameter $\beta_{6}$ represents the interaction effect of premise memory vividness, whereas $\beta_{7}$ indicates how that effect is modulated for threat trials compared to neutral.

\subsubsection{Effects of threat learning on premise memories}

Applying the same modelling strategy used to analyze the effect of threat learning and arousal on relational memory, we tested whether the original memories $(A \rightarrow B)$ were strengthened by 
the novel threat associations $(B \rightarrow C)$, which would be in line with earlier studies finding evidence of emotional tagging_(Dunsmoor et al., 2015; Zhu et al., 2020).

$$
\text { Odds_hit } t_{i j}=\mu+\alpha_{i}+\beta_{1}(\text { Threat })_{i j}+\beta_{2}(\text { Arousal })_{i j}+\beta_{3}(\text { Threat } \times \text { Arousal })_{i j}
$$

Note that now, Odds_hit ${ }_{i j,}$, refers to the binary outcome (correct or incorrect) of trials during the associative recognition test of associations from day one, on day three of the experiment.

Similarly, we tested whether the associations between novel memory elements $(B \rightarrow C)$ differed between threat trials and neutral controls. Here we expected an impairment in line with earlier studies towards the effect of emotion on associative learning (Bisby \& Burgess, 2014). 


\section{Results}

\subsection{Manipulation checks}

\subsubsection{Premise memories}

Following correction for premise memories for the main relational memory analysis, out of a total of 40 , an average of 33.66 trials $(S D=8.13$ ) remained for each participant (see Fig. $2 a)$. There was no statistically significant difference in how these were distributed across the two threat conditions ( $\left.t_{34}=1.13, p=0.198\right)$. Moreover, individual binomial tests revealed that each participant performed significantly above change on the relational memory test on premise corrected trials (chance level being $1 / 6$ correct).

\subsubsection{Pupil responses to C items and their predictors (B)}

To assess whether the aversive stimuli were successful at inducing physiological arousal, we first ran a $3 \times 2$ repeated measures ANOVA using learning Block and Condition as within-subject factors to investigate individuals' average pupil dilation to $\mathrm{C}$ stimuli (see Fig. $2 \mathrm{c}$ ). This revealed a main effect of Condition $\left(F_{1,31}=58.39, p=<0.001, \eta p^{2}=0.653\right)$. This is strong evidence that the aversive $\mathrm{C}$ stimuli indeed brought about a state of heightened arousal relative to the neutral stimuli. A main effect of Block $\left(F_{2,62}=15.27, p<0.001, \eta p^{2}=0.330\right)$ further implies that overall, pupil responsiveness decreased over time as participants performed the learning task. The absence of a significant Condition $\times$ Block interaction $\left(F_{2,62}=0.19, p=0.829\right)$ indicates that participants generally did not habituate to the aversive stimuli, as compared to the neutral stimuli, when these were presented for the second and third time. 
Then, to assess whether the emotional charge of ' $C$ ' items transferred to the neutral ' $B$ ' component of each trial, a repeated measures ANOVA with the same within-subject factors was conducted, now however to explain pupil dilation in response to B stimuli (see Fig. 2b). Critically, we found a significant interaction between learning Block and Condition $\left(F_{2,60}=9.55, p\right.$ $\left.<0.001, \eta p^{2}=0.241\right)$, indicating that anticipatory conditioned pupil responses develop over time and predictive learning has taken place. Planned comparisons show that ' $\mathrm{B}$ ' stimuli that predict aversive ' $C$ ' stimuli do not differ from ' $B$ ' stimuli that predict neutral ' $C$ ' stimuli during the first learning block $\left(t_{150}=-0.80, p=0.429\right)$, but do already elicit significantly greater pupil responses in the second block $\left(t_{150}=3.03, p=0.003\right)$, and this effect is even greater in the third learning block $\left(t_{150}=4.23, p<0.001\right)$. We again found main effects of both, Condition $\left(F_{1,30}=\right.$ 15.04, $p=0.005, \eta p^{2}=0.334$ ) implying generally larger anticipatory pupil responses preceding aversive events, and Block $\left(F_{2,60}=5.13, p=0.009, \eta p^{2}=0.146\right)$. These results confirm that the ' $C$ ' stimuli presented on the second day of the experiment were successful at evoking acute adrenergic responses, which during learning transferred to their associated conditioned ' $\mathrm{B}$ ' stimuli.

In line with these findings, participants' subjective self-reported affective responses to their own imagined stories on the second day differed greatly between conditions, with selfreported arousal being higher $\left(t_{34}=13.72, p<0.001\right.$, Cohen's $\left.d=2.46\right)$ and valence lower $\left(t_{34}=\right.$ 19.06, $p<0.001$, Cohen's $d=-3.42$ ) for threat trials compared to neutral trials. Together, these findings suggest that the present paradigm is suitable for investigating the respective effects of threat learning and acute noradrenergic arousal on relational memory. Moreover, the increase 
in arousal responses to $B$ items predictive of threat over learning blocks demonstrates that the episodic threat conditioning manipulation was successful.
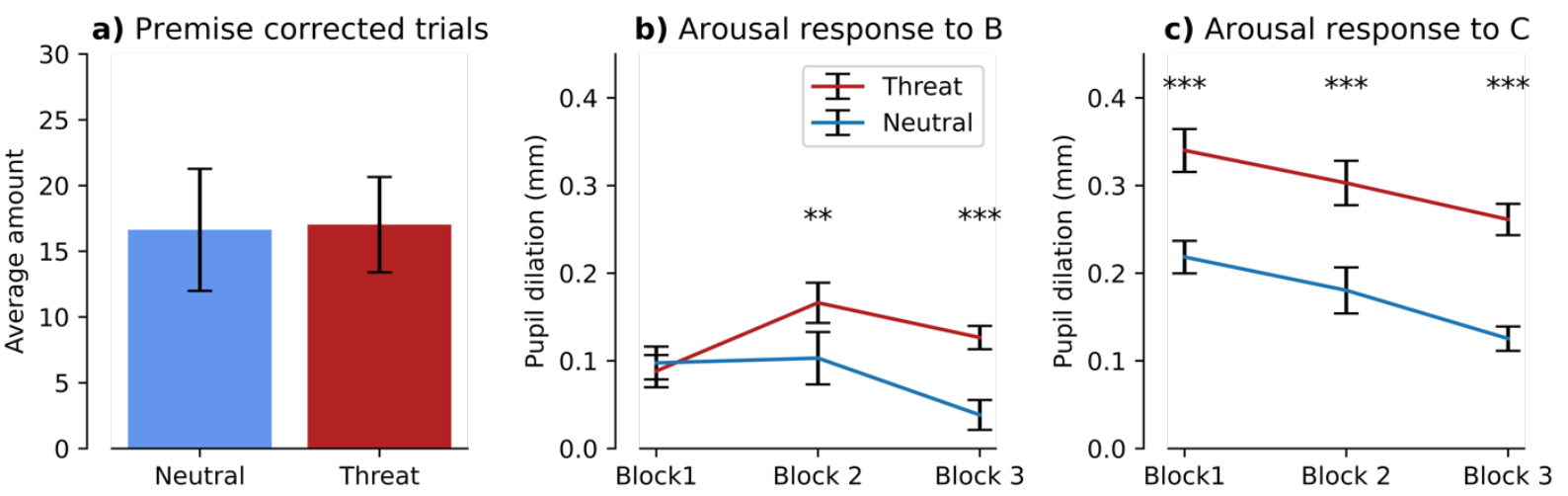

Fig 2. Analyses to validate the novel PRE-Memory paradigm. a) In total, participants correctly remembered both premise memories for an average of 33.66 out of 40 relational memory trials. These were divided equally across conditions. b) Pupil responses to neutral B stimuli that predicted an aversive or neutral C stimulus did not differ initially in block 1 , but diverged after the predictive associations were learned over the course of blocks 2 and 3, indicating successful threat acquisition. c) Pupil responses to the aversive $\mathrm{C}$ stimuli did not habituate - they remained consistently higher than the neutral ones throughout the experiment, even though pupil responsiveness gradually decreased over learning blocks. Error bars represent the standard error of the mean. Asterisks indicate statistically significant differences $\left({ }^{*} p<0.05,{ }^{* *} p<0.01\right.$, and $\left.{ }^{* * *} p<0.001\right)$.

\subsection{Primary analyses}

\subsubsection{Memories of threat learning impair relational memory}

To test the main hypothesis that threat learning memories affect subsequent relational memory judgments, we first ran a multilevel logistic regression model to estimate the effect of threat condition (categorical variable, neutral or threat) as the only predictor of correct or incorrect relational memory judgments. This revealed a significant negative effect of threat ( $\log$ odds $=$ - 
$\left.0.45, p=0.005, C l_{95}=[-0.773,-0.136]\right)$. This first analysis provides convincing evidence that threat impairs relational memory.

Table 1. Multilevel regression output corresponding to the analysis of the effect of threat learning on relational memory.

\begin{tabular}{llcl} 
Variable & Log odds & \multicolumn{1}{c}{ Cl95 $_{95}$} & $p$-value \\
\hline Intercept & 1.90 & {$[1.378,2.426]$} & $<\mathbf{0 . 0 0 1}$ \\
Threat & -0.45 & {$[-0.773,-0.136]$} & $\mathbf{0 . 0 0 5}$ \\
\hline
\end{tabular}

Note: $\mathrm{Cl}_{95}=95 \%$ confidence interval around the point estimate. Bold letters indicate significant $(<0.05)$ p-values.

A multilevel regression analysis of reaction times (RT) revealed that participants were faster $(\beta=$ $-8.083 \mathrm{~ms}, Z=-3.615, p<0.001)$ when making correct as compared to incorrect relational memory judgements. There was however no main effect of condition ( $\beta=1.020 \mathrm{~ms}, Z=0.839$, $p<0.402)$, nor an interaction effect of condition $\mathrm{x}$ correct on RTs $(\beta=-0.942 \mathrm{~ms}, Z=-0.698, p<$ 0.485), indicating no evidence for an accuracy-response time trade-off, nor did we observe evidence that participants avoided the threat trials by spending less time on their retrieval.

\subsubsection{Arousal does not drive the effect of threat}

We then analyzed whether initial arousal responses (operationalized here as pupil dilation) upon first encounter with an aversive $\mathrm{C}$ item, drive the impairment by memories of threat learning on relational memory. Including these variables in the model revealed a significant effect of arousal ( $\log$ odds $\left.=1.487, Z=2.43, p=0.015, C l_{95}=[-0.340,2.691]\right)$, and a marginally 
significant interaction of arousal and condition $\left(\log\right.$ odds $=-1.568, Z=-1.96, p=0.051, C_{95}=[-$ $2.855,0.163])$, on subsequent relational memory. Contrary to our hypothesis however, the positive odds ratio of arousal can be interpreted as arousal having an enhancing effect on relational memory for neutral trials. The negative odds ratio, of similar size, for the interaction $\mathrm{x}$ condition parameter indicates that this interaction is mostly driven by the neutral trials, while no effect for threat trials exists (see figure 3b). Indeed, there was no evidence for a relationship with arousal and relational memory for threat trials $\left(\log\right.$ odds $=0.081, Z=-0.16, p=0.870, C l_{95}=$ $[-0.081,0.496])$. The interpretation that threat condition cancels the enhancing effect of arousal was further confirmed by testing whether the hypothesis that subtracting the Condition $\mathrm{x}$ Arousal beta parameter from the Arousal parameter the equaled zero $\left(\chi^{2}(1)=0.017, p=0.896\right)$.

Table 2. Multilevel regression output when arousal is added to the model as a moderator.

\begin{tabular}{llll} 
Variable & Log odds & \multicolumn{1}{c}{ Cl $_{95}$} & $p$-value \\
\hline Intercept & 2.132 & {$[1.571,2.785]$} & $<\mathbf{0 . 0 0 1}$ \\
Threat & -0.548 & {$[-0.979,-0.216]$} & $\mathbf{0 . 0 0 8}$ \\
Arousal & 1.487 & {$[-0.340,2.691]$} & $\mathbf{0 . 0 1 5}$ \\
Threat x Arousal & -1.568 & {$[-2.855,0.163]$} & 0.051
\end{tabular}

Note: $\mathrm{Cl}_{95}=95 \%$ confidence interval around the point estimate. Bold letters indicate significant $(<0.05)$ p-values.
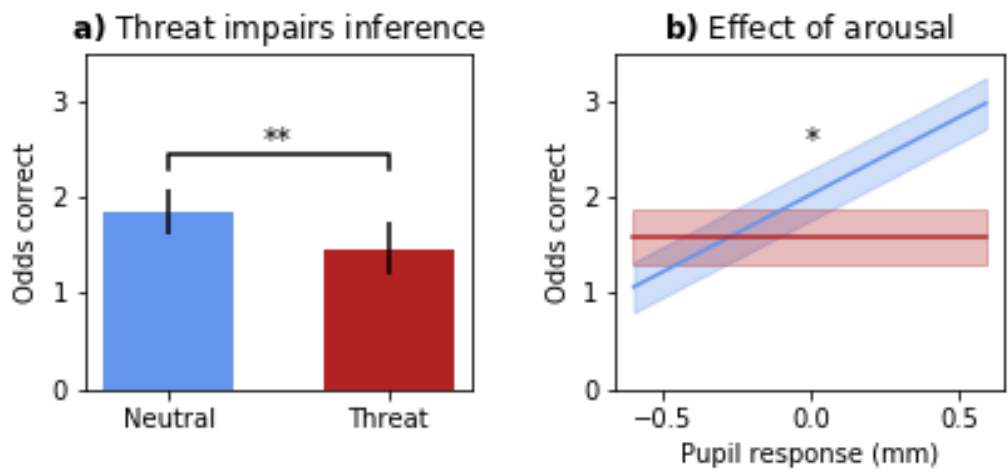
Fig 3. Results for each of the main hypotheses. Threat learning significantly impairs the odds of correct relational memory judgments (a). Furthermore, arousal induced by the $\mathrm{C}$ stimuli, here measured as a response in pupil dilation, had an enhancing effect on relational memory, but only for neutral trials (b). Error bars represent the standard error of the mean. Asterisks indicate statistically significant differences between conditions $\left({ }^{*} p<0.05\right.$, and $\left.{ }^{* *} p<0.01\right)$

\subsection{Secondary analyses}

\subsubsection{High vividness at encoding for both premise memories enhances relational}

\section{memory for neutral trials, but not threat trials}

We tested the potentially confounding role of vividness at encoding that may interact with threat learning in its subsequent effect on relational memory. The vividness scores for both days were added to the initial model of hypothesis 1 , allowing for every possible interaction with condition (Vividness day $1 \mathrm{x}$ vividness day $2 \mathrm{x}$ condition). The model showed no main effects or interaction with condition for vividness on either day one or two. However, there was a significant three-way interaction between condition, vividness on day 1, and vividness on day 2 $\left(\log\right.$ odds $\left.=-0.504, Z=-2.96, p=0.003, C l_{95}=[-0.837,-0.171]\right)$, indicating a difference between conditions in how relational memory is affected by vivid encoding when it is high on both days. To better interpret this result, we split the dataset by condition and ran the model again on both neutral and threat trials separately, with only vividness scores for both days as predictor variables. This revealed a statistically significant interaction term between vividness on day 1 and day 2 for neutral trials $\left(\log\right.$ odds $\left.=0.48, Z=3.0, p=0.003, C l_{95}=[0.169,0.79]\right)$, which was absent for the threat trials $\left(\log\right.$ odds $\left.=-0.07, Z=-0.730, p=0.466, C l_{95}=[-0.258,0.118]\right)$. These findings suggest that when all elements are neutral, relational memory is enhanced if both 
premise associations are vividly encoded, whereas trials that involve threat do not benefit from vividness in this manner (figure $3 c$ ).

Table 3. Multilevel regression output corresponding to the moderating role of premise memory vividness.

\begin{tabular}{llll} 
Variable & Log odds & \multicolumn{1}{c}{ Cl95 } & $p$-value \\
\hline Intercept & 1.947 & {$[1.394,2.500]$} & $<\mathbf{0 . 0 0 1}$ \\
Threat & -0.416 & {$[-0.753,-0.078]$} & $\mathbf{0 . 0 1 6}$ \\
Vividness_D1 & 0.238 & {$[-0.032,0.508]$} & 0.084 \\
Vividness_D2 & -0.039 & {$[-0.303,0.224]$} & 0.769 \\
Threat x Vividness_D1 & -0.143 & {$[-0.490,0.205]$} & 0.421 \\
Threat x Vividness_D2 & 0.239 & {$[-0.107,0.586]$} & 0.175 \\
Vividness_D1 x Vividness_D2 & 0.442 & {$[0.162,0.722]$} & $\mathbf{0 . 0 0 2}$ \\
Threat x Vividnesss_D1 x Vividness_D2 & -0.504 & {$[-0.837,-0.171]$} & $\mathbf{0 . 0 0 3}$ \\
\hline
\end{tabular}

Note: $\mathrm{Cl}_{95}=95 \%$ confidence interval around the point estimate. Bold letters indicate significant

$(<0.05)$ p-values.

\subsubsection{Original memories are enhanced following novel threat learning}

We tested the hypothesis that novel threat learning may strengthen associated elements of a pre-existing, reactivated memory, in line with earlier observations (Zhu et al., 2020). Moreover, we tested whether this effect was moderated by the anticipatory arousal responses that the neutral B items came to evoke following $B \rightarrow C$ learning, operationalized as the pupil response to $B$ during the third round of learning. Neutral associations that were later linked to an aversive item were significantly better retained on the final associative recognition test ( $\log$ odds $=$ $\left.0.454, p=0.016, C l_{95}=[0.085,0.827]\right)$. 
Table 4. Multilevel regression output showing the beneficial effect of threat learning on preexisting associative memories.

\begin{tabular}{llcl} 
Variable & Log odds & Cl95 $_{95}$ & $p$-value \\
\hline Intercept & 2.907 & {$[2.050,3.764]$} & $<\mathbf{0 . 0 0 1}$ \\
Threat & 0.454 & {$[0.085,0.827]$} & $\mathbf{0 . 0 1 6}$
\end{tabular}

Note: $\mathrm{Cl}_{95}=95 \%$ confidence interval around the point estimate. Bold letters indicate significant $(<0.05)$ p-values.

When arousal responses to B items in the third round of threat learning were added to the model, there were no significant effects (see table 5 below). It is however important to note that this model is based on far fewer trials than the condition-only model (758 as opposed to 1400 , nested in 24 participants out of 35) due to trials not meeting the quality criterion, and estimates two more parameters, and is likely underpowered as a result.

Table 5. Multilevel regression output showing no effects of arousal on premise memories

\begin{tabular}{llcl} 
Variable & Log odds & $C_{95}$ & $p$-value \\
\hline Intercept & 3.415 & {$[2.262,4.568]$} & $<\mathbf{0 . 0 0 1}$ \\
Threat & 0.022 & {$[-0.534,0.578]$} & 0.937 \\
Arousal & 0.397 & {$[-1.340,2.134]$} & 0.655 \\
Threat x Arousal & 0.190 & {$[-2.007,2.388]$} & 0.865
\end{tabular}

\subsubsection{No difference between threat learning and neutral associations}

Finally, we investigated whether there were differences in associative memory strength between the threat learning events and neutral controls. Particularly, we expected to find an impairment of threat condition (Bisby \& Burgess, 2014). However, no effect of threat condition was observed $\left(\log\right.$ odds $\left.=-0.235, p=0.402, C l_{95}=[-0.785,0.315]\right)$. 
Table 6. Multilevel regression output demonstrating no difference in strength between threatening and non-threatening associations.

\begin{tabular}{llll} 
Variable & Log odds & Cl95 $_{95}$ & $p$-value \\
\hline Intercept & 4.304 & {$[3.421,5.187]$} & $<\mathbf{0 . 0 0 1}$ \\
Threat & -0.235 & {$[-0.785,0.315]$} & 0.402
\end{tabular}

Note: $\mathrm{Cl}_{95}=95 \%$ confidence interval around the point estimate. Bold letters indicate significant $(<0.05)$ p-values.

Adding arousal to the model, operationalized as pupil responses to $C$ items in the first learning round of day two, again yielded no significant effects (see table 7). Note however that the log odds of the intercept in both models (4.304 and 4.508) is very high, corresponding to a hit-rate of $98 \%$ in the reference condition. This can be considered performance at ceiling, masking the potential effect of threat learning on associative recognition.

Table 7. Multilevel regression output demonstrating no effect of arousal on associative strength of day two memories

\begin{tabular}{llcl} 
Variable & Log odds & Cl $_{95}$ & $p$-value \\
\hline Intercept & 4.508 & {$[3.426,5.590]$} & $<\mathbf{0 . 0 0 1}$ \\
Threat & -0.483 & {$[-1.448,0.282]$} & 0.327 \\
Arousal & -0.575 & {$[-2.514,1.364]$} & 0.561 \\
Threat x Arousal & 0.300 & {$[-2.062,2.662]$} & 0.804
\end{tabular}

Note: $\mathrm{Cl}_{95}=95 \%$ confidence interval around the point estimate. Bold letters indicate significant $(<0.05)$ p-values. 


\section{Discussion}

To successfully navigate a complex world, it can be essential to be able to draw connections between memories of experiences that did not co-occur. Here we show that when an episodic memory of threat learning is part of a judgment that requires relational memory it actually hampers that ability, whereas the original neutral memory it overlaps with is retroactively strengthened. Unexpectedly, noradrenergic arousal did not further amplify these relationships. In fact, for neutral trials we observed a beneficial effect of arousal. Following this same pattern, high vividness of both premise memories was found to enhance the likelihood of an accurate relational memory judgement for neutral trials, but not threat trials. Together these results demonstrate that episodic threat learning not only hampers the subsequent recombination of elements across memories, but also nullifies the beneficial effects of noradrenergic arousal and premise memory vividness at time of encoding. This may hint at the existence of a mechanism that actively prevents the integration of emotional information with pre-existing neutral memories.

We hypothesized that relational memory would either be enhanced by episodic threat conditioning through prioritized integration, or impaired as a result of the mechanisms that have been proposed to hamper associative memory extending across-memories. The present findings are only consistent with the latter. Importantly, this impairment of episodic threat learning on relational memory cannot be explained as a resulting from an altered distribution of attention across stimuli while learning threatening associations as opposed to neutral controls for two reasons: First, stimuli to be associated were presented sequentially, as is a requirement for threat learning to occur. And second, by limiting our analysis to relational memory trials for 
which the premise memories were still retained on the final day of the experiment, we ruled out the possibility of the required premise memories never being properly encoded, or no longer retrievable on the day of testing. As such, the impairment of episodic threat learning on subsequent relational memory can only be ascribed to altered activity of mechanisms that are specifically relevant to the recombination of declaratively accessible information. The disruption hypothesis (Bisby et al., 2016) posits that the processing of emotional information overloads the hippocampus, causing a decrease in associative binding. The competing explanation by Madan and colleagues (2017), the bypassing hypothesis, instead attributes the hampering effect of emotion on associative memory to a decrease in extra-hippocampal processing typically involved in unitization. Relational memory, the ability to associate across experience, may have been impacted by either mechanism under the assumption that they operate similarly on the linkage between reactivated elements of memories and novel threat. However, neither hypothesis has been designed or tested for predictive and relational associations. Important questions thus remain pertaining to the nature of the representations underlying relational memory for predictive associations, and whether they are affected by threat before being required.

There are two explanations for the impairment by threat memories on relational memory within the memory integration framework. First, the negative effect can be due to emotional incongruence of the premise memories involved. A recent study showed that spatiocontextual similarity between encoding conditions of overlapping memories facilitates memory integration (Cox et al., 2021). In the present study, threat condition may have functioned in a comparable manner as an internal encoding context. Neutral control memories on day two 
were of the same valence as their reactivated counterparts from day one, whereas memories of threat learning, which were encoded in a state of heightened arousal, were not, potentially resulting in disrupted integration (Clewett et al., 2020). A second possibility is that, rather than passively impairing integration, threat learning actively pushes overlapping memories towards the opposite, namely representational differentiation. Several neuroimaging studies of memory integration surprisingly found a decrease of representational similarity for overlapping as compared to non-overlapping memory traces, and little is currently understood about the circumstances under which either integration, pattern separation, or differentiation occurs (For review, see Brunec et al., 2020). The results presented here could be explained as threat prediction being a factor that promotes differentiation of memories. This also accounts for the finding that, in line with the results of Zhu and colleagues (2020), newly acquired predictive value retroactively enhanced the associative strength of neutral premise memories from day one, well after the initial consolidation window had passed (Schafe \& LeDoux, 2000). If novel memories of threat learning and the memories they overlap with are actively differentiated, those pre-existing memories would become more representationally distinct relative to pattern separated controls, and hence more resilient to interference. We thus propose that an emotion induced enhancement of associative binding within overlapping memories is compatible with, and possibly results from, differentiation between them. If emotion retroactively stimulates the associative binding of within-premise memory elements whilst pushing towards differentiation of the overlap, this changes the availability of strategies for relational memory between conditions (Shohamy \& Daw, 2015). For neutral relational memory judgements then, one may either draw on a readily available integrated representation or piece together the relations by 
retrieving both memories at moment it is required, whereas for those involving memories of threat learning, the luxury of a pre-made map of all elements will be less common. Functional magnetic resonance imaging combined with multivoxel pattern analysis will be required to test the hypothesis that hippocampal differentiation of memories mediates the hampering effect of episodic threat learning on relational memory.

Contrary to our prediction, we did not find the effect of episodic threat learning on relational memory to be graded as a function of stimulus-induced noradrenergic arousal. This is somewhat surprising given that arousal has consistently been implicated as the dimension driving the detrimental effect of emotion on associative memory. This discrepancy can perhaps be explained due to differences in paradigms, or mechanisms underlying direct associative and relational memory. However, in this literature, arousal has rarely been operationalized physiologically and analyzed on a trial-by-trial basis, rather than aggregated by subject. It is possible that its effect, and in particular our understanding thereof, has been overstated as a result. Furthermore, for neutral trials we found a positive effect of arousal. The reason for this interaction may be that variance in pupil dilation, thought to reflect a summation of multiple psychological constructs (Mathôt, 2018), was driven by different proportions of latent factors across conditions. Many participants indicated difficulties complying with instructions specifically during threat trials, as they were frequently overwhelmed by their content and consequently unable to stick with the task of imagining stories involving them. This is supported by the high drop-out rate on day two of $21.3 \%$ due to the aversive stimuli. Pupil dilation responses in the neutral condition may thus reflect a combination of arousal, effort, and elaboration that is beneficial to relational memory, whereas the latter two key ingredients were 
likely absent from the equation in the threat condition. Similarly, variance in vividness ratings may too be subject to different interpretations across conditions: beneficial anchoring to prior knowledge for neutral trials (Fujiwara et al., 2021), and vivid sensory processing for threat trials (Murray \& Kensinger, 2013).

In conclusion, we here reveal that memories of threat learning hamper subsequent relational memory. Even though at first sight it may be particularly adaptive to connect past neutral memories with related threat learning events, humans may be protected against such 'episodic overgeneralization' of threat by a mechanism that compartmentalizes, rather than integrates, negative experiences, causing impairments in relational memory. This however raises the question whether this mechanism is to some degree defective in clinical populations. Our memories form the foundation for our predictions and simulations of the future (Schacter et al., 2007), and if these are based on a memory system that does not protect itself from needlessly integrating episodic threat memories into its web of connections, the result could be maladaptive rumination and anxiety. The present study shows that a healthy memory may actively prevent the unnecessary linkage of reactivated elements to threat, thus potentially safeguarding against such symptoms. 


\section{References}

Biderman, N., Bakkour, A., \& Shohamy, D. (2020). What Are Memories For? The Hippocampus Bridges Past Experience with Future Decisions. Trends in Cognitive Sciences, 24(7), 542-556. https://doi.org/10.1016/j.tics.2020.04.004

Bisby, J. A., \& Burgess, N. (2014). Negative affect impairs associative memory but not item memory. Learning and Memory, 21(1), 21-27. https://doi.org/10.1101/Im.032409.113

Bisby, J. A., Horner, A. J., Bush, D., \& Burgess, N. (2018). Negative emotional content disrupts the coherence of episodic memories. Journal of Experimental Psychology: General, 147(2), 243-256. https://doi.org/10.1037/xge0000356

Bisby, J. A., Horner, A. J., Hørlyck, L. D., \& Burgess, N. (2016). Opposing effects of negative emotion on amygdalar and hippocampal memory for items and associations. Social Cognitive and Affective Neuroscience, 11(6), 981-990. https://doi.org/10.1093/scan/nsw028

Bradley, M. B., Miccoli, L. M., Escrig, M. a, \& Lang, P. J. (2008). The pupil as a measure of emotional arousal and automatic activation (Author Manuscript). Psychophysiology, 45(4), 602. https://doi.org/10.1111/j.14698986.2008.00654.x.The

Brodeur, M. B., Dionne-Dostie, E., Montreuil, T., \& Lepage, M. (2010). The bank of standardized stimuli (BOSS), a new set of 480 normative photos of objects to be used as visual stimuli in cognitive research. PLoS ONE, 5(5). https://doi.org/10.1371/journal.pone.0010773

Brogden, W. J. (1947). Sensory preconditioning of human subjects. Journal of Experimental Psychology, 37(6), 527539. https://doi.org/10.1037/h0058465

Brunec, I. K., Robin, J., Olsen, R. K., Moscovitch, M., \& Barense, M. D. (2020). Integration and differentiation of hippocampal memory traces. Neuroscience and Biobehavioral Reviews, 118(March), 196-208. https://doi.org/10.1016/j.neubiorev.2020.07.024

Chiu, Y. C., Dolcos, F., Gonsalves, B. D., \& Cohen, N. J. (2013). On opposing effects of emotion on contextual or relational memory. Frontiers in Psychology, 4(MAR), 2-5. https://doi.org/10.3389/fpsyg.2013.00103

Clewett, D., Gasser, C., \& Davachi, L. (2020). Pupil-linked arousal signals track the temporal organization of events 
in memory. Nature Communications, 11(1), 1-14. https://doi.org/10.1038/s41467-020-17851-9

Cox, W. R., Dobbelaar, S., Meeter, M., Kindt, M., \& Ast, V. A. Van. (2021). Episodic memory enhancement versus impairment is determined by contextual similarity across events. PsyArXiv. https://psyarxiv.com/w7csx/

Dunsmoor, J. E., Murty, V. P., Davachi, L., \& Phelps, E. A. (2015). Emotional learning selectively and retroactively strengthens memories for related events. Nature, 520(7547), 345-348. https://doi.org/10.1038/nature14106

Eichenbaum, H. (1999). The hippocampus and mechanisms of declarative memory. Behavioural Brain Research, 103(2), 123-133. https://doi.org/10.1016/S0166-4328(99)00044-3

Ellenbogen, J. M., Hu, P. T., Payne, J. D., Titone, D., \& Walker, M. P. (2007). Human relational memory requires time and sleep. Proceedings of the National Academy of Sciences of the United States of America, 104(18), 7723-7728. https://doi.org/10.1073/pnas.0700094104

Fujiwara, E., Madan, C. R., Caplan, J. B., \& Sommer, T. (2021). Emotional arousal impairs association memory : roles of prefrontal cortex regions. 76-82. https://doi.org/10.1101//m.052480.120.28

Guez, J., Saar-Ashkenazy, R., Mualem, L., Efrati, M., \& Keha, E. (2015). Negative emotional arousal impairs associative memory performance for emotionally neutral content in healthy participants. PLOS ONE, 10(7), 114. https://doi.org/10.1371/journal.pone.0132405

Hernandez, P. J., \& Abel, T. (2009). The role of protein synthesis in memory consolidation: Progress amid decades of debate. Neurobiology of Learning and Memory, 89(3), 293-311. https://doi.org/10.1016/j.nlm.2007.09.010.The

Klein, S. B., Cosmides, L., Tooby, J., \& Chance, S. (2002). Decisions and the evolution of memory: Multiple systems, multiple functions. Psychological Review, 109(2), 306-329. https://doi.org/10.1037/0033-295X.109.2.306

Koenig, S., Uengoer, M., \& Lachnit, H. (2017). Pupil dilation indicates the coding of past prediction errors: Evidence for attentional learning theory. In Psychophysiology (Vol. 55, Issue 4). Psychophysiology. https://doi.org/10.1111/psyp.13020

Krypotos, A. M., Effting, M., Arnaudova, I., Kindt, M., \& Beckers, T. (2014). Avoided by association: Acquisition, extinction, and renewal of avoidance tendencies toward conditioned fear stimuli. Clinical Psychological Science, 2(3), 336-343. https://doi.org/10.1177/2167702613503139 
LaBar, K. S., \& Cabeza, R. (2006). Cognitive neuroscience of emotional memory. Nature Reviews Neuroscience, 7(1), 54-64. https://doi.org/10.1038/nrn1825

Liao, H. I., Kidani, S., Yoneya, M., Kashino, M., \& Furukawa, S. (2016). Correspondences among pupillary dilation response, subjective salience of sounds, and loudness. Psychonomic Bulletin and Review, 23(2), 412-425. https://doi.org/10.3758/s13423-015-0898-0

Madan, C. R., Fujiwara, E., Caplan, J. B., \& Sommer, T. (2017). Emotional arousal impairs association-memory: Roles of amygdala and hippocampus. Neurolmage, 156(May), 14-28. https://doi.org/10.1016/j.neuroimage.2017.04.065

Marchewka, A., Żurawski, Ł., Jednoróg, K., \& Grabowska, A. (2014). The Nencki Affective Picture System (NAPS): Introduction to a novel, standardized, wide-range, high-quality, realistic picture database. Behavior Research Methods, 46(2), 596-610. https://doi.org/10.3758/s13428-013-0379-1

Mathôt, S. (2018). Pupillometry: Psychology, Physiology, and Function. Journal of Cognition, 1(1), 1-23. https://doi.org/10.5334/joc.18

McClelland, J. L., McNaughton, B. L., \& O'Reilly, R. C. (1995). Why there are complementary learning systems in the hippocampus and neocortex: Insights from the successes and failures of connectionist models of learning and memory. Psychological Review, 102(3), 419-457. https://doi.org/10.1037/0033-295X.102.3.419

McGaugh, J. L. (2004). The amygdala modulates the consolidation of memories of emotionally arousing experiences. Annual Review of Neuroscience, 27(1), 1-28. https://doi.org/10.1146/annurev.neuro.27.070203.144157

McGaugh, J. L. (2018). Emotional arousal regulation of memory consolidation. Current Opinion in Behavioral Sciences, 19, 55-60. https://doi.org/10.1016/j.cobeha.2017.10.003

Murray, B. D., \& Kensinger, E. A. (2012). The effects of emotion and encoding strategy on associative memory. Memory and Cognition, 40(7), 1056-1069. https://doi.org/10.3758/s13421-012-0215-3

Murray, B. D., \& Kensinger, E. A. (2013). A review of the neural and behavioral consequences for unitizing emotional and neutral information. Frontiers in Behavioral Neuroscience, 7(APR 2013), 1-12. https://doi.org/10.3389/fnbeh.2013.00042

Schacter, D. L., Addis, D. R., \& Buckner, R. L. (2007). Remembering the past to imagine the future: The prospective 
brain. Nature Reviews Neuroscience, 8(9), 657-661. https://doi.org/10.1038/nrn2213

Schafe, G. E., \& LeDoux, J. E. (2000). Memory consolidation of auditory pavlovian fear conditioning requires protein synthesis and protein kinase A in the amygdala. The Journal of Neuroscience: The Official Journal of the Society for Neuroscience, 20(18), 1-5. https://doi.org/10.1523/jneurosci.20-18-j0003.2000

Schlichting, M. L., Mumford, J. A., \& Preston, A. R. (2015). Learning-related representational changes reveal dissociable integration and separation signatures in the hippocampus and prefrontal cortex. Nature Communications, 6, 1-10. https://doi.org/10.1038/ncomms9151

Shohamy, D., \& Daw, N. D. (2015). Integrating memories to guide decisions. Current Opinion in Behavioral Sciences, 5, 85-90. https://doi.org/10.1016/j.cobeha.2015.08.010

Shohamy, D., \& Wagner, A. D. (2008). Integrating Memories in the Human Brain: Hippocampal-Midbrain Encoding of Overlapping Events. Neuron, 60(2), 378-389. https://doi.org/10.1016/j.neuron.2008.09.023.Integrating

Stevenson, Ryan A ; James, T. W. (2008). Affective auditory stimuli : Characterization of the International ... Behavior Research Methods, 40(1), 315-321. https://doi.org/10.3

Todd, R. M., Talmi, D., Schmitz, T. W., Susskind, J., \& Anderson, A. K. (2012). Psychophysical and neural evidence for emotion-enhanced perceptual vividness. Journal of Neuroscience, 32(33), 11201-11212. https://doi.org/10.1523/JNEUROSCI.0155-12.2012

Tolman, E. C. (1948). Cognitive maps in rats and men. The Psychological Review, 55(4), 189-208. https://doi.org/10.4324/9780203789155-11

Willenbockel, V., Sadr, J., Fiset, D., Horne, G. O., Gosselin, F., \& Tanaka, J. W. (2010). Controlling low-level image properties: The SHINE toolbox. Behavior Research Methods, 42(3), 671-684.

https://doi.org/10.3758/BRM.42.3.671

Wimmer, G. E., \& Shohamy, D. (2012). Preference by Association : Science, 338(October), $270-273$. https://doi.org/10.1126/science.1223252

Zeithamova, D., Dominick, A. L., \& Preston, A. R. (2013). Hippocampal and ventral medial prefrontal activation during retrieval-mediated learning supports novel inference. Neuron, 75(1), 168-179. https://doi.org/10.1016/j.neuron.2012.05.010.Hippocampal

Zeithamova, D., Schlichting, M. L., \& Preston, A. R. (2012). The hippocampus and inferential reasoning: building 
memories to navigate future decisions. Frontiers in Human Neuroscience, 6(March), 1-14.

https://doi.org/10.3389/fnhum.2012.00070

Zhu, Z., Y., Y., Zhang, Z., L., L., Zeng, Z., Y., Y., Chen, C., C., C., Fernandez, F., G., G., Qin, Q., \& S., S. (2020). Emotional tagging retroactively promotes memory integration through rapid neural reactivation and reorganization. BioRxiv, 2020.09.09.285890.

http://biorxiv.org/cgi/content/short/2020.09.09.285890v1?rss=1\&utm_source=researcher_app\&utm_mediu m=referral\&utm_campaign=RESR_MRKT_Researcher_inbound 